\title{
Regulatory network-based imputation of dropouts in single-cell RNA sequencing data
}

Ana Carolina Leote1,5, , Xiaohui $\mathrm{Wu}^{1,3,{ }^{*}, \text { Andreas Beyer } 1,2,4,5, \#}$

1. Cologne Excellence Cluster on Cellular Stress Responses in Aging-Associated Diseases (CECAD), University of Cologne, 50923 Cologne, Germany

2. Cologne School for Computational Biology \& Center for Data Science and Simulation, University of Cologne, 50923 Cologne, Germany

3. Department of Automation, Xiamen University, Xiamen 361005, China

4. Center for Molecular Medicine of the University of Cologne, 50931 Cologne, Germany

5. University of Cologne, Faculty of Medicine and University Hospital Cologne

${ }^{*}$ Equal contribution.

\# Corresponding author:

Phone: +49-221-478 84429

Fax: +49-221-478 84026

E-Mail: andreas.beyer@uni-koeln.de 


\section{Abstract}

Single-cell RNA sequencing (scRNA-seq) methods are typically unable to quantify the expression levels of all genes in a cell, creating a need for the computational prediction of missing values ('dropout imputation'). Most existing dropout imputation methods are limited in the sense that they exclusively use the scRNA-seq dataset at hand and do not exploit external gene-gene relationship information.

Here, we show that a transcriptional regulatory network learned from external, independent gene expression data improves dropout imputation. Using a variety of human scRNA-seq datasets we demonstrate that our network-based approach outperforms published state-of-the-art methods. The network-based approach performs particularly well for lowly expressed genes, including cell-typespecific transcriptional regulators. Additionally, we tested a baseline approach, where we imputed missing values using the sample-wide average expression of a gene. Unexpectedly, up to $48 \%$ of the genes were better predicted using this baseline approach, suggesting negligible cell-to-cell variation of expression levels for many genes. Our work shows that there is no single best imputation method; rather, the best method depends on gene-specific features, such as expression level and expression variation across cells. We thus implemented an R-package called ADImpute (available from https://github.com/anacarolinaleote/ADImpute) that automatically determines the best imputation method for each gene in a dataset. 


\section{Introduction}

Single-cell RNA sequencing (scRNA-seq) has become a routine method, revolutionizing our understanding of biological processes as diverse as tumor evolution, embryonic development, and ageing. However, current technologies still suffer from the problem that large numbers of genes remain undetected in single cells, although they actually are expressed (dropout events). Although dropouts are enriched among lowly expressed genes, relatively highly expressed genes can be affected as well. Of course, the dropout rate is also dependent on the sampling depth, i.e. the number of reads or transcript molecules (UMIs) quantified in a given cell. Imputing dropouts is necessary for fully resolving the molecular state of the given cell at the time of the measurement. In particular, genes with regulatory functions - e.g. transcription factors, kinases, regulatory ncRNAs - are typically lowly expressed and hence particularly prone to be missed in scRNA-seq experiments. This poses problems for the interpretation of the experiments if one aims at understanding the regulatory processes responsible for the transcriptional makeup of the given cell.

A range of computational methods have been developed to impute dropouts using the expression levels of detected genes. The underlying (explicit or implicit) assumption is often that detected and undetected genes are subject to the same regulatory processes, and hence detected genes can serve as a kind of 'fingerprint' of the state at which the cell was at the time of lysis. Several popular methods are based on some type of grouping (clustering) of cells based on the similarity of their expression patterns. Missing values are then imputed as a (weighted) average across those similar cells where the respective gene was detected ${ }^{1-4}$. For example, the MAGIC algorithm ${ }^{1}$ creates a network of cells by linking cells with similar gene expression signatures. Missing values are subsequently imputed by computing an average over linked cells, where cells get weighted based on how similar or dissimilar their expression signatures are compared to the target cell. DrImpute ${ }^{3}$ and scImpute ${ }^{2}$ have further developed this notion and have been shown to outperform MAGIC in recent comparisons ${ }^{5}$. These methods rest on two important assumptions: (1) the global expression pattern of a cell (i.e. across the subset of detected genes) is predictive for all genes; (2) the (weighted) average of co-clustering (i.e. similar) cells is a good estimator of the missing value. The first assumption is violated if the expression of a dropout gene is driven by only a small subset of genes and hence the global expression pattern does not accurately reflect the state of the relevant sub-network. Any global similarity measure of the whole transcriptome will be dominated by the majority of genes ${ }^{5}$. The second assumption is violated if the data is scarce, i.e. when either only few similar cells were measured or if the particular gene was detected in only a small subset of cells. In that case the average is computed across a relatively small number of observations and hence unstable. 
bioRxiv preprint doi: https://doi.org/10.1101/611517; this version posted June 10,2020. The copyright holder for this preprint (which was not certified by peer review) is the author/funder, who has granted bioRxiv a license to display the preprint in perpetuity. It is made available under aCC-BY-NC 4.0 International license.

The recently published SAVER ${ }^{6}$ method employs a different strategy that can overcome some of these limitations. SAVER attempts to predict the posterior probability distribution for the 'true' expression of each gene in each cell. This distribution is derived from the data by learning gene-gene relationships from the dataset, which are subsequently used to predict the gene- and cell-specific probability distribution of the expected expression value. In other words, SAVER does not use the whole transcriptome of a cell to predict the expression level of a given gene; instead, it uses a specific subset of genes that are expected to be predictive for the particular gene at hand.

SCRABBLE is different compared to all of the other methods mentioned above, because it can use bulk sequencing data to assist in the imputation. SCRABBLE combines a de-noising step with a moderated imputation moving the sample means towards the observed (bulk-derived) mean expression values.

Here, we compare published approaches that are representative for current state-of-the-art methods to two fundamentally different approaches. The first is a very simple baseline method that we use as a reference approach: we estimate missing values as the average of the expression level of the given gene across all cells in the dataset where the respective gene was detected. Initially intended to serve just as a reference for minimal expected performance, this sample-wide averaging approach turned out to perform surprisingly well and in many instances even better than state-of-the-art methods. The simple explanation is that estimating the average using all cells is a much more robust estimator of the true mean than using only a small set of similar cells, especially when the gene was detected in only few cells and/or if the gene's expression does not vary much across cells.

The second new approach avoids using a global similarity measure comparing entire transcriptomes. Instead, similar to SAVER it rests on the notion that genes are part of regulatory networks and only a small set of correlated or functionally associated genes should be used to predict the state of undetected genes. However, unlike SAVER, we propose to use transcriptional regulatory networks trained on independent (bulk seq) data to rigorously quantify the transcriptional relationships between genes. Missing values are then imputed using the expression states of linked genes in the transcriptional regulatory network and exploiting the known quantitative relationships between genes. This approach allows imputing missing states of genes even in cases where the respective gene was not detected in any cell or in only extremely few cells. This second new approach rests on the assumption that the network describes the true regulatory relationships in the cells at hand with sufficient accuracy. Here, we show that this is indeed the case and that combining the two new approaches with published state-of-the-art methods drastically improves the imputation of scRNAseq dropouts. Importantly, the performance of an imputation method is dependent on the 'character' of a gene (e.g. its expression level or the variability of expression between cells). Hence, we 
bioRxiv preprint doi: https://doi.org/10.1101/611517; this version posted June 10,2020. The copyright holder for this preprint (which was not certified by peer review) is the author/funder, who has granted bioRxiv a license to display the preprint in perpetuity. It is made available under aCC-BY-NC 4.0 International license.

implemented an R-package (Adaptive Dropout Imputer, or ADImpute) that determines the best imputation method for each gene through a cross-validation approach.

\section{Results}

\section{Imputing dropouts using a transcriptional regulatory network}

In order to understand whether the inclusion of external gene regulatory information allows for more accurate scRNA-seq dropout imputation, we derived a regulatory network from bulk gene expression data in 1,376 cancer cell lines with known karyotypes. For this purpose, we modelled the change (compared to average across all samples) of each gene as a function of its own copy number state and changes in predictive genes:

$$
y_{i}=\alpha_{i} \cdot c_{i}+\sum_{j \neq i} \alpha_{i j} \cdot y_{j}+\varepsilon_{i},
$$

where $y_{i}$ is the expression deviation (log fold change) of gene $i$ from the global average, $c_{i}$ is the known (measured) copy number state of gene $i, \alpha$ the vector of regression coefficients, $y_{j}$ the observed change in expression of gene $j$ and $\varepsilon_{i}$ the i.i.d. error of the model. To estimate a set of predictive genes $j$, we made use of LASSO regression ${ }^{7}$, which penalizes the L1 norm of the regression coefficients to determine a sparse solution. LASSO was combined with stability selection ${ }^{8}$ to further restrict the set of predictive genes to stable variables and to control the false discovery rate (Methods). Using the training data, models were fit for 24,641 genes, including 3,696 non-coding genes. The copy number state was only used during the training of the model, since copy number alterations are frequent in cancer and can influence the expression of affected genes. If copy number states are known, they can of course also be used during the dropout imputation phase. Using cell line data for the model training has the advantage that the within-sample heterogeneity is much smaller than in tissue-based samples ${ }^{9}$. However, in order to evaluate the general applicability of the model across a wide range of conditions, we validated its predictive power on a diverse set of tissuebased bulk-seq expression datasets from the The Cancer Genome Atlas (4,548 samples from 13 different cohorts; see Methods and Supplementary Figs. 1-2) and the Genotype-Tissue Expression (17,382 samples from 30 different healthy tissues; see Methods and Supplementary Figs. 3-4).

Such a model allows us to estimate the expression of a gene that is not quantified in a given cell based on the expression of its predictors in the same cell. Here, the difficulty lies in the fact that imputed dropout genes might themselves be predictors for other dropout genes, i.e. the imputed expression of one gene might depend on the imputed expression of another gene. In order to derive the 
imputation scheme based on the model from equation (1), we revert to an algebraic expression of the problem,

$$
Y=A Y,
$$

where $A$ is the adjacency matrix of the transcriptional network, with its entries $\alpha_{i j}$ being fitted using the regression approach described above, and $Y$ is the vector of gene expression deviations from the mean across all cells in a given cell. In the current implementation we assume no copy number changes and hence, we exclude the $c_{i}$ term from equation (1). Like in equation (1), we omit the intercept since we are predicting the deviation from the mean. Subsequently, imputed values are recentered using those means to shift imputed values back to the original scale (see Methods). Further note that we drop the error term $\varepsilon$ from equation (1), because this is now a prediction task (and not a regression). Here, we exclusively aim to predict dropout values, and (unlike SAVER) our goal is not to improve measured gene expression values. Hence, measured values remain unchanged. It is therefore convenient to further split $Y$ into two sub-vectors $Y^{m}$ and $Y^{n}$, representing the measured and non-measured expression levels, respectively. Likewise, $A$ is reduced to the rows corresponding to non-measured expression levels and split into $A^{m}$ (dimensionality $|n| \times|m|$ ) and $A^{n}$ (dimensionality $|n| \times|n|$ ), accounting for the contributions of measured and non-measured genes, respectively. The imputation problem is then reduced to:

$$
Y^{n}=A^{n} Y^{n}+A^{m} Y^{m}
$$

As $Y^{m}$ is known (measured) and will not be updated by our imputation procedure, the last term can be condensed in a fixed contribution, $F=A^{m} Y^{m}$, accounting for measured predictors:

$$
Y^{n}=A^{n} Y^{n}+F
$$

The solution $Y^{n}$ for this problem is given by:

$$
Y^{n}=\left(I-A^{n}\right)^{-1} F
$$

The matrix $\left(I-A^{n}\right)$ may not be invertible, or if it is invertible, the inverse may be unstable. Therefore, we computed the pseudoinverse $\left(I-A^{n}\right)^{+}$using the Moore-Penrose inversion. Computing this pseudoinverse for every cell is a computationally expensive operation. Thus, we implemented an additional algorithm finding a solution in an iterative manner (Methods). Although this iterative second approach is not guaranteed to converge, it did work well in practice (see Supplementary Fig. 5 , Methods). While our R-package implements both approaches, subsequent results are based on the iterative procedure. 


\section{Transcriptional regulatory network information improves scRNA-seq dropout imputation}

To assess the performance of our network-based imputation method and compare it to that of previously published methods, we considered three different single-cell RNA sequencing datasets (Supplementary Table 1). The first dataset focuses on human embryonic stem cell (hESC) differentiation and comprises 1,018 cells - hESCs, lineage progenitors derived from hESCs and human foreskin fibroblasts ${ }^{10}$. Cell type labels are known for this dataset. A second dataset comprises 4,347 cells from 6 human oligodendrogliomas ${ }^{11}$. Finally, data from healthy pancreata of 2 human donors $^{12}$ were also used for test purposes. It was important to include a range of different healthy cell types in the evaluation, because the transcriptional regulatory network was trained on cancer cell line data. Thus, by including data from non-cancerous tissues, we could evaluate possible restrictions induced by the model training data.

In order to quantify the performance of both proposed and previously published imputation methods, we randomly set a fraction of the quantified values in the test data to zero according to two different schemes (Methods) and stored the original values for later comparison with the imputed values. Imputation was then performed on the masked dataset using our network-based approach, DrImpute ${ }^{3}$, SAVER$^{6}$, scImpute ${ }^{2}$ and SCRABBLE ${ }^{13}$. Those methods were chosen since they were shown to be among the top-performing state-of-the-art dropout imputation methods ${ }^{14}$. As a baseline method, the masked and actual dropout values were assigned the average $\log _{2}$-transformed normalized expression across all cells where the corresponding gene was quantified. For masked entries imputed by all tested methods, the original and imputed values were compared to determine an imputation error per gene (Methods).

As expected, imputation error increased with increasing missing information (NAs) per gene in the data (Fig. 1, Supplementary Fig. 6). While this is true for practically all methods, scImpute (Fig. 1, turquoise line) was particularly sensitive to missing information about genes across cells, a behaviour also described elsewhere ${ }^{14}$. A similar trend was observed for DrImpute (Fig. 1, green line), which also borrows information from similar cells for dropout imputation. SCRABBLE, which takes into account bulk gene expression levels, outperformed scImpute and DrImpute with increasing missing genes. Of note, the performance of SCRABBLE was better when using a pseudo-bulk reference derived from averaging all cells in the dataset together (Fig. 1, purple lines, dashed), as compared to using external bulk RNA-seq expression data (Fig. 1, hESC differentiation, purple line, full). Only within the ranges of very rarely detected genes was the use of bulk RNA-seq data beneficial. Our network-based approach outperformed all previously published methods (Fig. 1). As the network-based approach uses information regarding other genes contained in the same cell, we 
hypothesized its accuracy might be more affected by increasingly sparse information per cell when compared to other methods. However, although the average imputation error slightly increased with the number of missing genes per cell, this was true for all methods and the relative performance differences were largely invariant to the number of missing genes per cell (Supplementary Fig. 7). Further, the network-based method performed well over a range of different cell types and showed decreased performance upon randomization of the transcriptional network (Methods, Supplementary Fig. 8). Thus, the diversity of cell lines used in the training data seemed to capture a large fraction of all possible regulatory relationships in the human transcriptome.

The performance of the Baseline method (Fig. 1, grey line), which does not account for any expression variation between cells, was surprising to us. While SAVER showed a poor performance in comparison to all other methods (also described in ${ }^{14}$ ), it should be noted that this method aims to estimate the true value for all genes, not only for the dropout genes. Hence, its goal is slightly different from that of the other methods in this comparison.

Baseline and Network can impute missing values for many more genes than all other methods tested here (Supplementary Table 3). In order to also evaluate the quality of those method-specific imputations, we repeated the performance evaluation considering all masked values (Supplementary Fig. 9). Under this scheme, we observed that the relative performance of the different methods was largely maintained, apart from a decrease in the performance of SCRABBLE without bulk RNA-seq information.

Further, as an alternative way of assessing the performance of the imputation methods, we quantified the correlation between the original values before masking and the results of each imputation procedure (Supplementary Table 2, Supplementary Fig. 12). As opposed to computing the residuals between imputed and measured values, the regression is independent of mean shifts in the predictions. We observed the same relative performance of the methods as with the imputation error analysis. Taken together, these results indicate that both the Baseline and the network-based approach often lead to more accurate and numerous (Fig. 1, Supplementary Table 3) imputations than state-of-the-art imputation methods. 
hESC differentiation

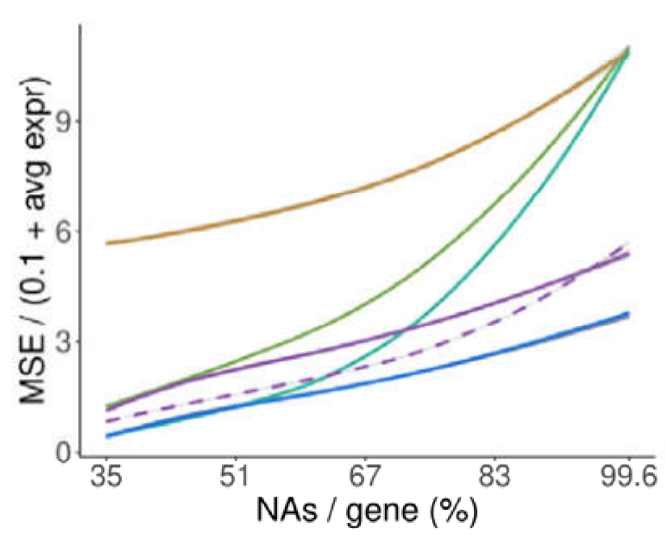

Healthy pancreas (indiv. 1)

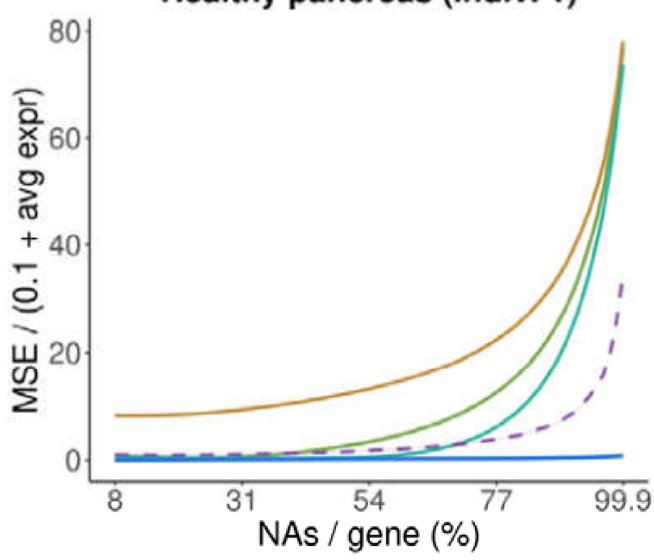

\section{Oligodendroglioma}

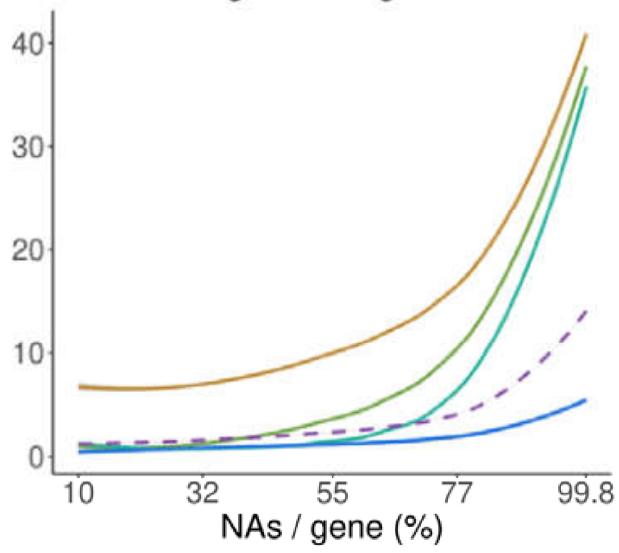

Healthy pancreas (indiv. 4)

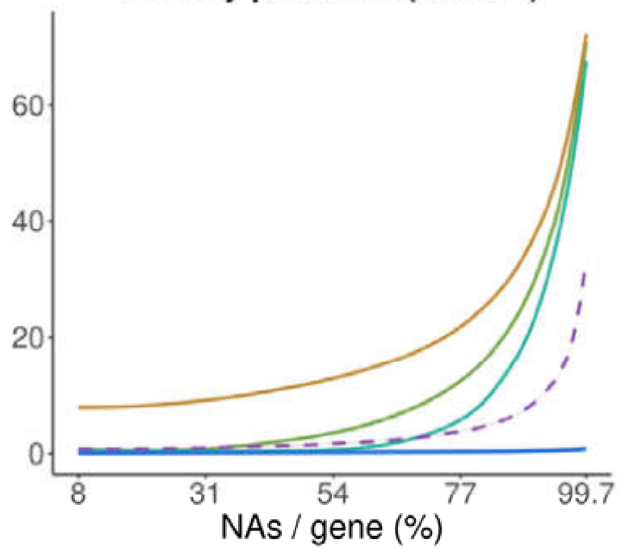

$$
\begin{array}{llll}
\text { - Baseline } & \text {-SAVER } & \text {-SCRABBLE, bulk } & \text { - Network } \\
\text { - Drlmpute } & \text { - sclmpute } & \text { - SCRABBLE, sc ref } &
\end{array}
$$

Figure 1: Imputation error of Baseline (slate grey), DrImpute (green), SAVER (yellow), scImpute (turquoise), SCRABBLE (purple) - with available bulk data as reference (full line) or the average of the single cell data as reference (dashed line) - and Network (blue). Loess trendline of weighted Mean Squared Error (MSE) of imputation with percentage of missing values per gene, for all 4 test datasets, restricted to values that could be imputed by all seven methods. The trend line for Baseline is below that of Network. Note that even though in this analysis Network and Baseline show the same average behavior, the performance on individual genes can differ considerably.

Table 1: Percentage of genes best imputed by each method (lowest weighted MSE) in the four test datasets restricted to values that could be imputed by all six methods.

\begin{tabular}{|c|c|c|c|c|}
\hline & $\begin{array}{c}\text { hESC } \\
\text { differentiation }\end{array}$ & Oligodendroglioma & $\begin{array}{c}\text { Healthy pancreas } \\
\text { (indiv. 1) }\end{array}$ & $\begin{array}{c}\text { Healthy pancreas } \\
\text { (indiv. 4) }\end{array}$ \\
\hline Baseline & $33.6 \%$ & $48.1 \%$ & $10.6 \%$ & $13.7 \%$ \\
\hline DrImpute & $0.4 \%$ & $0.2 \%$ & $0.1 \%$ & $0.1 \%$ \\
\hline SAVER & $0.1 \%$ & $0 \%$ & $0 \%$ & $0 \%$ \\
\hline scImpute & $21.1 \%$ & $4.1 \%$ & $0.2 \%$ & $0.2 \%$ \\
\hline SCRABBLE, bulk & $4.8 \%$ & - & - & - \\
\hline SCRABBLE, sc ref & $5.5 \%$ & $1.7 \%$ & $0.3 \%$ & $0.2 \%$ \\
\hline Network & $34.5 \%$ & $45.8 \%$ & $88.9 \%$ & $85.7 \%$ \\
\hline
\end{tabular}


bioRxiv preprint doi: https://doi.org/10.1101/611517; this version posted June 10,2020. The copyright holder for this preprint (which was not certified by peer review) is the author/funder, who has granted bioRxiv a license to display the preprint in perpetuity. It is made available under aCC-BY-NC 4.0 International license.

\section{Gene features determine the best performing imputation method}

To characterize the genes best imputed by each of the methods, we determined, for each gene in each test dataset, the method resulting in the lowest weighted Mean Squared Error of imputation (Table 1). The genes best imputed by each method were then compared against a background including all genes imputed by all four methods (Fig. 2 and Supplementary Fig. 13). As expected, rarely detected genes were best imputed by the Network and Baseline methods. In particular, genes with low expression and low variance were best imputed by the Baseline, while Network was able to perform the best imputations for genes across a wide range of expression levels and variance. Methods relying on the similarity of cellular transcriptomes (scImpute, DrImpute and SCRABBLE) performed best for moderately expressed, more frequently detected genes. Notably, as data sparsity increased, so did the advantage in performance of Network and Baseline over the remaining methods (Fig. 1, Table 1, Supplementary Fig. 13).
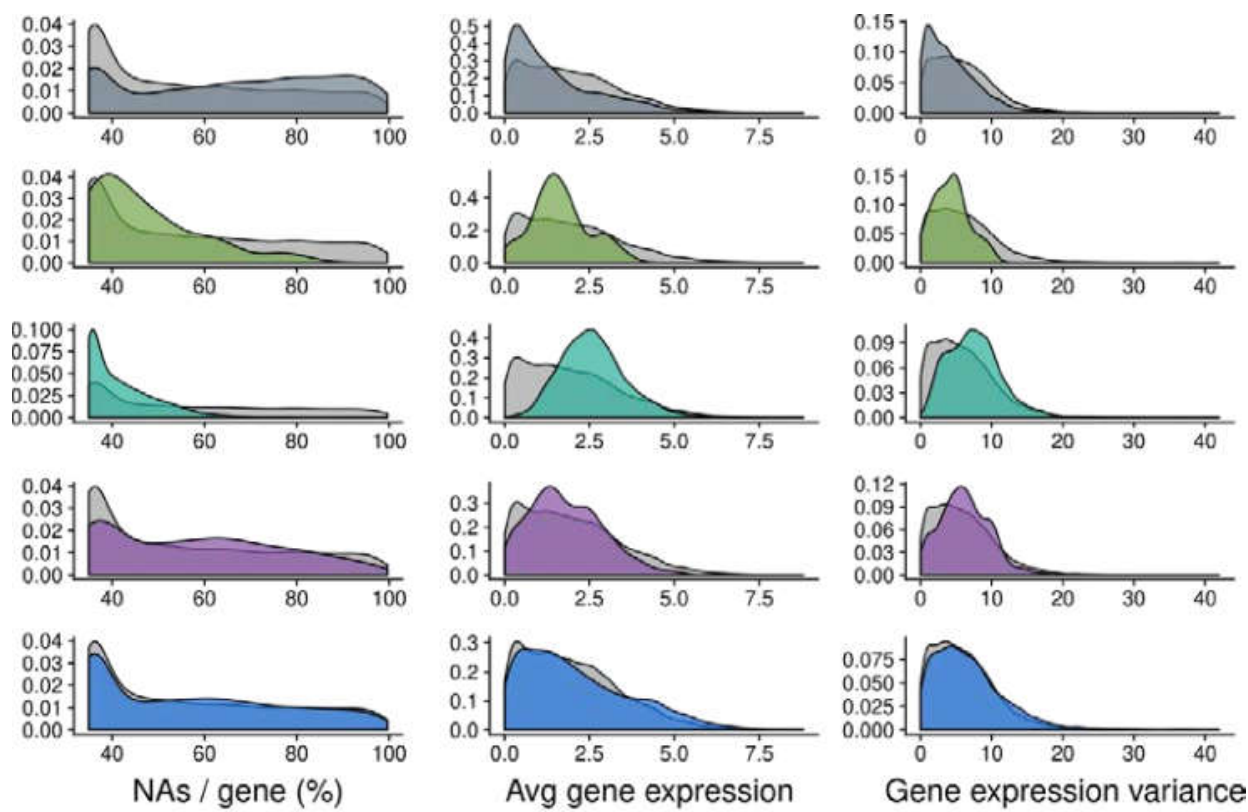

Background $\square$ Baseline

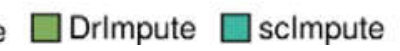

$\square$ SCRABBLE $\square$ Network

Figure 2: Characterization of the genes best predicted by the methods Baseline (slate grey), DrImpute (green) scImpute (turquoise), SCRABBLE (average of single cell data as reference; purple) and Network (blue) in the hESC differentiation dataset. Distribution of missing values per gene, average expression levels and variance of the genes best predicted by Baseline, scImpute and Network methods, compared against all tested genes (background). Due to the very low numbers of genes best imputed by SAVER, the corresponding distributions are not shown. Average gene expression is shown as $\log _{2}$-transformed normalized expression. 
bioRxiv preprint doi: https://doi.org/10.1101/611517; this version posted June 10,2020. The copyright holder for this preprint (which was

\section{Network-based imputation uncovers cluster markers and regulators}

A popular application of scRNA-seq is the identification of discrete sub-populations of cells in a sample in order to, for example, identify new cell types. The clustering of cells and the visual 2D representation of single-cell data is affected by the choice of the dropout imputation method 13 . Therefore, we assessed the impact of dropout imputation on data visualization using Uniform Manifold Approximation and Projection (UMAP) ${ }^{15}$ on the hESC data before and after imputation by all methods. The hESC dataset was particularly suitable in this case, because it was of high quality and it consisted of six well-annotated cell types. This analysis confirmed that the choice of the imputation method impacts on the grouping/clustering of cells (Supplementary Fig. 14).

We next asked to what extent the detection of cluster markers would be affected by the choice of the imputation method. Thus, we applied Seurat ${ }^{16}$ to the hESC differentiation dataset, which was composed of a well-defined set of distinct cell types, before and after imputation. We then defined genes that were significantly differentially expressed between one cluster and all the others as cluster markers (Methods). We observed a strong overlap between markers detected before and after applying the tested imputation methods (Fig. 3A, rightmost bar, Supplementary Fig. 15), suggesting a common core of detected cluster markers across methods. Additionally, the numbers of significant markers detected after Network and Baseline imputations were lower than for other imputation methods (Fig. 3B). Imputation with scImpute and, to a smaller extent, with DrImpute, led to the highest number of significant markers (Fig. 3B). We hypothesized that many of these marker genes may result from artefactual clustering of cells. In order to test that notion we first determined all GO biological process terms that were enriched in the respective cell clusters without any dropout imputation. We termed them 'high confidence GO terms' since they are independent of the choice of the imputation method. It turned out that scImpute and DrImpute had the weakest enrichments in high confidence GO biological process terms (Fig. 3C-D; Methods; Supplementary Table 4), suggesting that the extra markers found upon applying scImpute and DrImpute contained many false positives, which diluted biological signals. Conversely, Network and SCRABBLE led to the strongest enrichments in high confidence GO biological process terms (Fig. 3C-D). 
A)

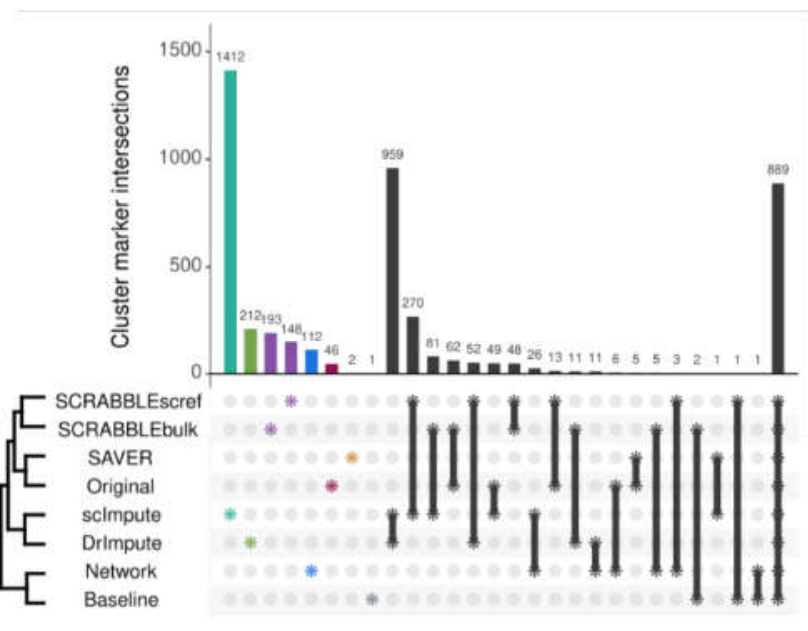

C)

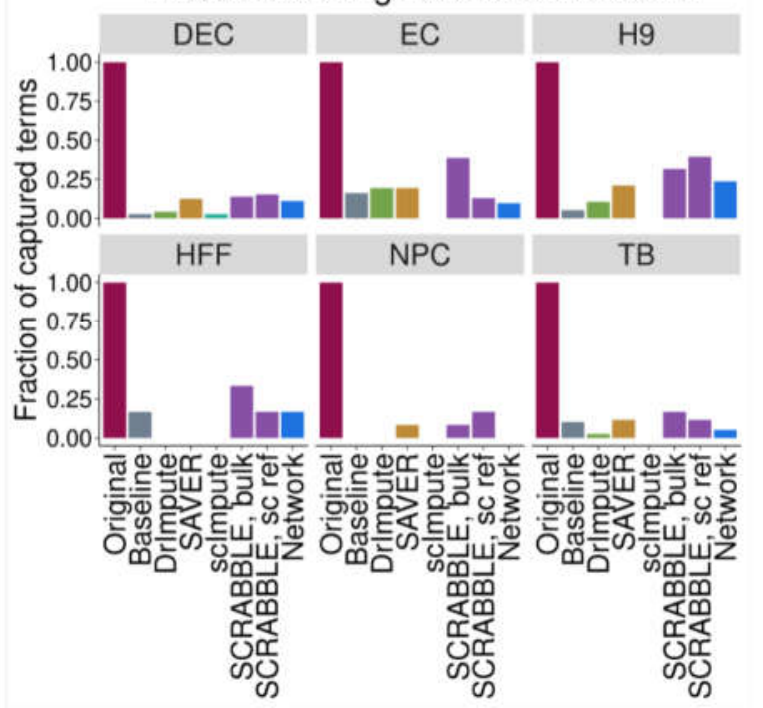

B)

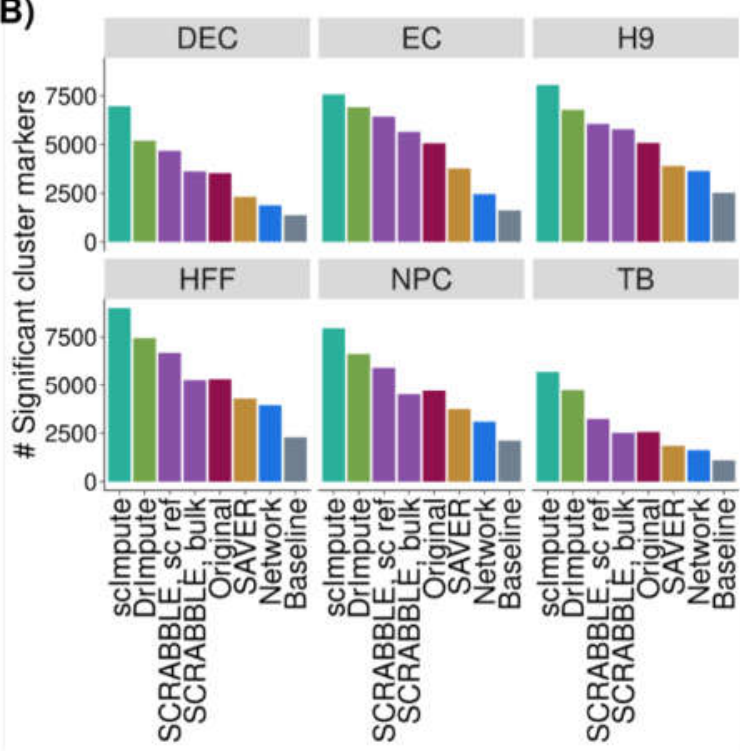

D)

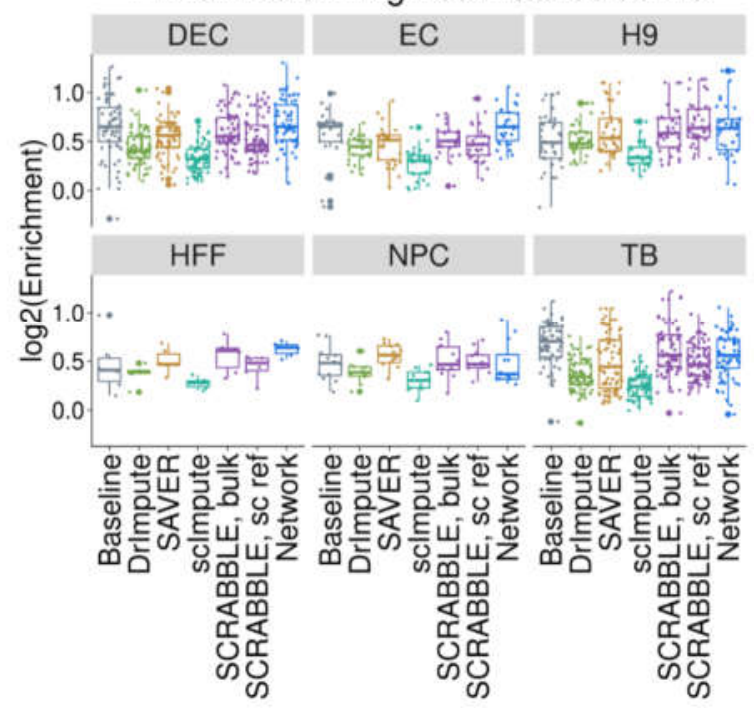

Figure 3: Detection of cell type-specific markers before and after imputation. A) Overlap between significant (FDR < $0.05,\left|\log _{2} \mathrm{FC}\right|>0.25$ ) definitive endoderm cell markers detected with no dropout imputation (Original) and using the tested imputation methods. B) Number of significant cell type markers detected with no dropout imputation and using the tested imputation methods. C) and D) fraction of captured high confidence terms, defined as significantly enriched (p.value $<0.001$ and $\log _{2}$ Enrichment $>0.5$, Methods) GO biological process terms among the cluster markers detected without imputation. C) Fraction of high confidence terms detected as significantly enriched (p.value $<0.001$ and $\log _{2}$ Enrichment $>0.5$ ) among the cluster markers detected with each imputation method. D) $\log _{2}$-enrichment of all high confidence terms among the cluster markers detected with each imputation method. DEC: definitive endoderm cells; EC: endothelial cells; H9: undifferentiated human embryonic stem cells; HFF: human foreskin fibroblasts; NPC: neural progenitor cells; TB: trophoblast-like cells.

Genes with regulatory functions are particularly important for understanding and explaining the transcriptional state of a cell. However, since genes with regulatory functions are often lowly 
expressed 17 , they are frequently subject to dropouts. Since our analysis had shown that the networkbased approach is especially helpful for lowly expressed genes (Fig. 2), we hypothesized that the imputation of transcript levels of regulatory genes would be particularly improved. In order to test this hypothesis, we further characterized those cluster markers that were exclusively detected using the network-based method. Indeed, we observed regulatory genes to be enriched among those markers (Fig. 4A). Among these markers exclusively detected upon network-based imputation, the transcription factor EHF was the second most significant trophoblast-specific. EHF is a known epithelium-specific transcription factor that has been described to control epithelial differentiation ${ }^{18}$ and to be expressed in trophoblasts ${ }^{19}$, even though at very low levels (EHF expression found among the first quintile of bulk TB RNA-seq data from the same authors). While EHF transcripts were not well captured in TB single-cell RNA-seq data (only quantified in 39 out of 775 TB cells), a trophoblastspecific expression pattern was recovered after network-based imputation (Fig. 4B, upper panel), but not with any of the other tested imputation methods (Supplementary Fig. 16). Similarly, OSR1 has been described as a relevant fibroblast-specific transcription factor ${ }^{20}$ which failed to be detected without imputation. Imputing with Network lead to the strongest fibroblast-specific expression pattern of OSR1 (Fig. 4B, lower panel), (Supplementary Fig. 16). Interestingly, TWIST2 and PRRX1, described by Tomaru et al.20 to interact with OSR1, also showed fibroblast-specific expression (Supplementary Fig. 17). Taken together, these results suggest that imputation based on transcriptional regulatory networks can recover the expression levels of relevant, lowly expressed regulators affected by dropouts. 
A)

Enrichment in "molecular function regulator" GO term

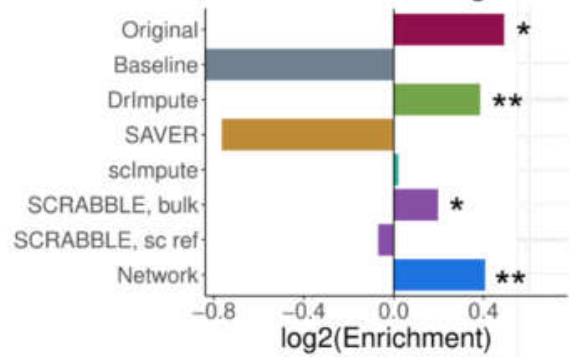

B)

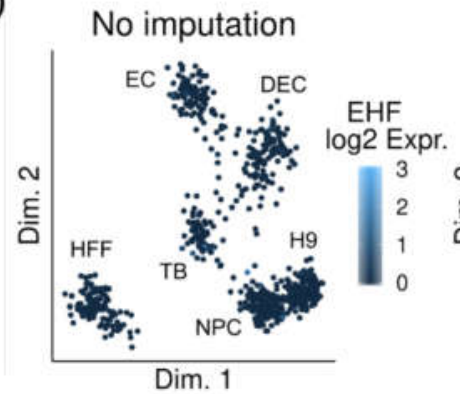

Network imputation

No imputation
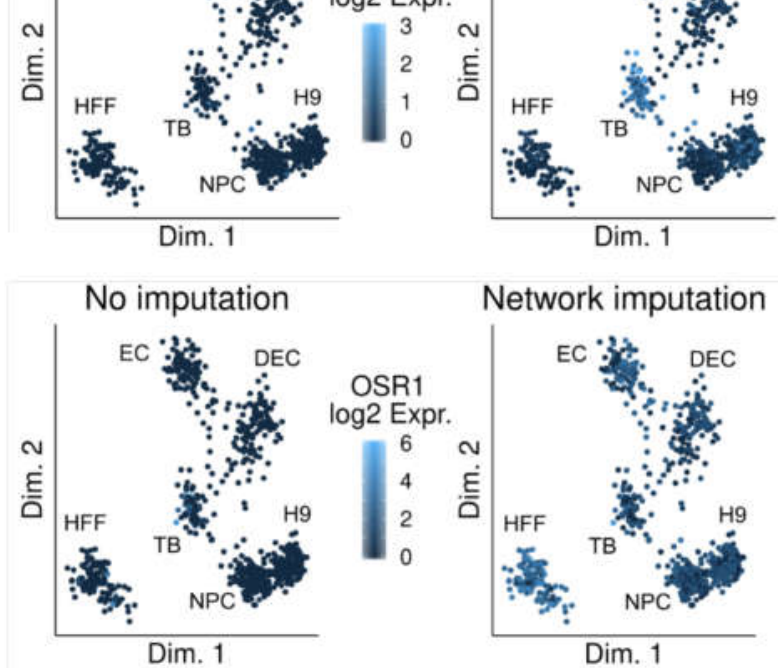

Network imputation

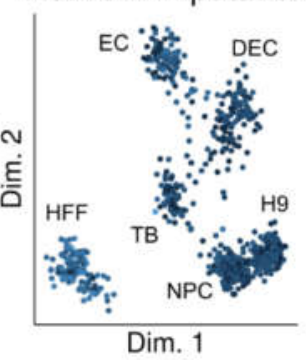

Figure 4: Detection of cell type-specific transcription factors is improved upon network-based imputation. A) Enrichment score in GO term "molecular function regulator" among the genes uniquely detected after each imputation approach. **: p-val < 0.01; *: p-val < 0.05. B) Projection of cells onto a low dimension representation of the data before imputation, using ZINB-WaVe ${ }^{21}$. Color represents normalized expression levels of EHF (top) and OSR1 (bottom) before and after Network-based imputation. DEC: definitive endoderm cells; EC: endothelial cells; H9: undifferentiated human embryonic stem cells; HFF: human foreskin fibroblasts; NPC: neural progenitor cells; TB: trophoblast-like cells. 


\section{Discussion}

A first important and surprising finding of our analysis is the fact that the sample-wide average expression performs well for the imputation of many genes (Table 1). As expected, genes whose expression levels were best imputed by this method were characterized by lower variance across cells and by remaining undetected in relatively many cells (Fig. 2). A potential problem of methods based on co-clustering cells is that the number of observations per cluster can get very small, which makes the estimation of the true mean more unstable. Thus, the average using all cells is preferred when the gene was detected in only few cells and/or if the gene's expression does not vary much across cells. Further, our findings imply that cell-to-cell variation of gene expression is negligible for many genes.

Second, the consideration of external gene co-expression information for the dropout imputation substantially improved the performance in many cases, especially for lowly expressed genes. Since genes with regulatory functions are often lowly expressed ${ }^{17}$, imputation of those genes might be critical for explaining expression variation between cells. Network can be seen as an extension of Baseline, because it predicts the cell-specific deviation from the population mean. This explains the often similar performance of the two methods.

The seemingly poor performance of SAVER can at least in part be attributed to our evaluation scheme, which is based on masking observed values. SAVER performs a re-scaling of predicted mean values (i.e. the means of the posterior distributions) that leads to larger deviations from the masked values. Another potential problem is the fact that SAVER learns gene-gene relationships from the scRNA-seq data itself, which may be imprecise or even impossible for genes with many dropouts. SAVER may very well maintain relative differences between genes and it clearly has the advantage of predicting the most likely true expression values also for observed genes (a feature not shared with any of the other methods evaluated in this work). Thus, one needs to choose the imputation method based on the specific goals. A potential limitation of our approach is that a transcriptional network derived from bulk-seq data may not fully capture gene-gene relationships that are detectable from single cell data. For example, gene regulatory relationships that are specific to a small sub-population of cells in a bulk tissue may not be correctly captured, because the signal would be too weak. A second example would be genes regulated during the cell cycle. Bulk tissue is usually not synchronized, i.e. it consists of a mix of cells at different cell cycle stages, which may prevent the detection of those relationships. To some extent these limitations were alleviated by using cell line data rather than actual tissue data for training the network. Of course, the network that we used here is still imperfect. However, despite that imperfection it demonstrated the power of our approach. Using it was clearly advantageous over not using it in most cases. 
The third -- and maybe most important -- conclusion is that the best performing imputation method is gene- and dataset-dependent. That is, there is no single best performing method. If the number of observations is high (many cells with detected expression) and if the expression quantification is sufficiently good, scImpute and DrImpute outperformed other methods. Importantly, the technical quality of the quantification depends on the read counts, which in turn depends on gene expression, transcript length and mappability - i.e. multiple factors beyond expression. If however, gene expression is low and/or too imprecise, scImpute and DrImpute were outcompeted by other methods. This finding led us to conclude that a combination of imputation methods would be optimal. Hence, we developed an R-package that determines 'on the fly' for each gene the best performing imputation method by masking observed values (i.e. via cross validation). This approach has the benefit that it self-adapts to the specificities of the dataset at hand. For example, the network-based approach might perform well in cell types where the assumptions of the co-expression model are fulfilled, whereas it might fail (for the same gene) in other cell types, where these assumptions are not met. Hence, the optimal imputation approach is gene- and dataset-dependent. An adaptive method selection better handles such situations. Another benefit of this approach is that the cross validation error can be used as a quantitative guide on how 'imputable' a given gene is in a specific scRNA-seq dataset. We have therefore implemented and tested this approach (see Supplementary Fig. 18). The resulting R-package (called ADImpute) is open to the inclusion of future methods, includes scImpute's estimation of dropout probability and it can be downloaded from http://cellnet.cecad.uni-koeln.de/adimpute.

We believe that this work presents a paradigm shift in the sense that we should no longer search for the single best imputation approach. Rather, the task for the future will be to find the best method for a particular combination of gene and experimental condition.

\section{Methods}

\section{Pre-processing of cancer cell line data for transcriptional regulatory network inference}

Entrez IDs and corresponding gene symbols were retrieved from the NCBI (https://www.ncbi.nlm.nih.gov/gene/?term=human\%5Borgn\%5D). Genome annotation was obtained from Ensembl (Biomart). Finally, genes of biotype in protein coding, ncRNA, snoRNA, scRNA, snRNA were used for network inference. For CCLE22, 768 cell lines that were used in Seifert et al. ${ }^{9}$ were used. Raw CEL files were downloaded from https://portals.broadinstitute.org/ccle/ and processed using the $\mathrm{R}$ package RMA in combination with a BrainArray design file (HGU133Plus2_Hs_ENTREZG_21.0.0). Final expression values were in $\log _{2}$ scale. Expression levels 
and CNV data set from RNA-seq were downloaded from Klijn et al.23. Before combining, each dataset is $\log _{2}$ transformed and scaled to $(0,1)$ for all genes in each sample using $\mathrm{R}$ function scale. Then datasets were merged and the function ComBat from the sva R package ${ }^{24}$ was used to remove batch effect of the data source. The final combined data set contains 24641 genes in 1443 cell lines. Finally, expression levels of genes were subtracted by the average expression level across all cell lines of the corresponding gene.

\section{Network inference based on stability selection}

The network inference problem can be solved by inferring independent gene-specific sub-networks. We used the linear regression model from equation (1) to model the change in a target gene as dependent on the combination of the gene-specific CNA and changes in all other genes. Here the intercept is not included because the data is assumed to be centered. We used LASSO with stability selection $^{8}$ to find optimal model parameters $\alpha_{i j}$.

The R package stabs was employed to implement stability selection and the glmnet package was used to fit the generalized linear model. Two parameters regarding error bounds were set with the cutoff value being 0.6 and the per-family error rate being 0.05 . A set of stable variables were defined by LASSO in combination with stability selection. Then coefficients of the selected variables were estimated by fitting generalized linear models using the R function $\mathrm{glm}$.

\section{Network validation using TCGA and GTEx data}

Gene expression and gene copy number data of 14 different tumor cohorts (4548 tumor patients in total) from TCGA collected in a previous study ${ }^{9}$ were used for validation. We examined the predictive power of our inferred networks on each TCGA cohort by predicting the expression level of each gene for each tumor using the corresponding copy number and gene expression data.

Additionally, in order to validate the applicability of the learnt network to healthy tissues, we further leveraged gene expression data from the Genotype-Tissue Expression (GTEx) Project. Read counts were downloaded from the portal website (version 8), normalized using the R package DESeq2 and centered gene-wise across tissues.

For each TCGA cohort or GTEx tissue, the expression levels of each gene were predicted using the network and expression quantification of the interacting genes in the same sample. The predicted value was then compared to the observed value, present in the original dataset. The quality of prediction for each TCGA cohort or GTEx tissue was quantified as either the correlation between predicted and observed expression of a gene across all samples or the MSE of prediction of a gene 
across all samples. A strong positive correlation or high MSE for a gene suggests high predictive power by the network on the respective gene.

\section{Single-cell test data processing}

Human embryonic stem cell differentiation data ${ }^{10}$ were downloaded from the Gene Expression Omnibus (GEO, accession number GSE75748) in the format of expected counts. Only snapshot singlecell data were used in this work (file GSE75748_sc_cell_type_ec.csv.gz). The downloaded data were converted to RPM (reads per million). Oligodendroglioma data ${ }^{11}$ were downloaded from GEO (accession number GSE70630) as $\log _{2}(\mathrm{TPM} / 10+1$ ) and converted back to TPM. Healthy human pancreas data ${ }^{12}$ were downloaded from GEO (accession number GSE84133) as UMI counts and converted to RPM.

\section{Dropout imputation}

Version 0.0.9 of scImpute ${ }^{2}$ was used for dropout imputation, in "TPM" mode for the oligodendroglioma dataset and "count" mode for all other datasets, without specifying cell type labels. The parameters were left as default, except for drop_thre $=0.3$, as the default of 0.5 resulted in no imputations performed. Cell cluster number (Kcluster) was left at the default value of 2 for imputation of the oligodendroglioma and healthy pancreata datasets and set to 6 for the hESC differentiation dataset, in order to match the number of cell clusters identified by the authors ${ }^{10}$. SAVER 1.1.1 was used with size.factors $=1$. SCRABBLE 0.0.1 was run with the parameters suggested by the authors and using by default the average gene expression across cells as the bulk reference. In the case of the hESC differentiation dataset, bulk data from the same study was available, and thus was used as reference. For all other imputation methods, the data was log2-transformed with a pseudocount of 1. DrImpute 1.0 was run using the default parameters. For dropout imputation by average expression ('Baseline'), gene expression levels were log2-transformed with a pseudocount of 1 and the average expression of each gene across all cells, excluding zeros, was used for imputation. For network-based imputation, expression values were log2-transformed with a pseudocount of 1 and centered gene-wise across all cells. The original centers were stored for posterior re-conversion. Subsequently, cell-specific deviations of expression levels from those centers were predicted using either equation (5) or the following iterative procedure. During the iteration genes were first predicted using all measured predictors. Subsequently, genes with dropout predictors were repredicted using the imputed values from the previous iteration. This was repeated for at most 50 iterations. The obtained values were added to the gene-wise centers. We note that, while the values after imputation cannot be interpreted as TPMs/RPMs, as the sum of the expression levels per sample 
is no longer guaranteed to be the same across samples. However, one could still perform a new normalization by total signal (sum over all genes) to overcome this issue.

\section{Masking procedures}

In order to compare the imputation error of the tested methods, we randomly masked (set to zero) some of the values for each gene, using two different approaches.

The first approach consisted of setting a fraction of the quantified, uniformly sampled values to zero for each gene (Fig. 1) - 35\% for the hESC differentiation dataset, 10\% for the oligodendroglioma dataset and 8\% for both healthy pancreas datasets. In case of Supplementary Fig. 7 30\% of the cells (not genes) were sampled. This unbiased masking scheme is in agreement with previous work ${ }^{25}$. The differing percentages of masked values per gene in each dataset result in a comparable sparsity of the data (around 84\% missing values) after masking.

As an alternative masking procedure that represents more closely a downsampling process, we modelled for each gene its probability to be an observed zero in the following way: the fraction of cells where each gene was not captured (zero in the original data) was modelled as a function of its average expression across cells (Supplementary Fig. 10). For this, a cubic spline was used, with knots at each $10 \%$ quantile of the average expression levels, excluding the $0 \%$ and $100 \%$ quantiles. A cubic spline was chosen so that it could properly fit to both UMI-based and non UMI-based datasets. With this model, a 'dropout probability' $p$ was computed for each gene from its mean expression. The masking procedure then consisted of, for each entry, sampling a Bernoulli distribution with probability of success 1- $p$, where 0 corresponds to a mask (the entry is set to 0 ) and 1 to leaving the data as it is. Thus, each entry in the data matrix may be masked with a probability $p$, which is genespecific and based on the observed dropout rates in the dataset at hand.

We observed the same relative performance of the imputation methods under this alternative masking scheme (Supplementary Fig. 11), and for this reason present the results obtained with the first masking approach.

\section{Imputation error analysis}

Imputation was performed with each of the four tested methods separately and the imputed masked entries were then compared to the original ones. As dropout-specific performance measure, we used the squared imputation error corrected for average gene expression (log-transformed normalized expression):

$$
\frac{(\text { original-imputed })^{2}}{\text { avg expression }+0.1}
$$


bioRxiv preprint doi: https://doi.org/10.1101/611517; this version posted June 10,2020. The copyright holder for this preprint (which was not certified by peer review) is the author/funder, who has granted bioRxiv a license to display the preprint in perpetuity. It is made available under aCC-BY-NC 4.0 International license.

The weighting prevents that the average error is dominated by highly expressed genes, i.e. some weighting is necessary. Another alternative would have been to just divide by the average expression (without adding 0.1). In that case however, the error estimates would have been heavily dominated by very lowly expressed genes since their average is very close to zero. Thus, the weighting proposed here is a fair compromise considering both, highly and lowly expressed genes.

\section{Dimensionality reduction and marker detection}

Dimensionality reduction on the original hESC differentiation data (Fig. 4B) was performed using ZINB-WaVe, implemented in the R package zinbwave ${ }^{21}$. H1 and TB cells in Batch 3 were removed to avoid confounding batch effects and, for the remaining cells, 2 latent variables were extracted from the information contained in the top 1000 genes with highest variance across cells. Batch information and the default intercepts were included in the ZINB-WaVe model, using epsilon $=1000$. K-means clustering $(\mathrm{k}=6)$ on the 2 latent variables strongly matched the annotated cell type labels $(0.977$ accuracy), confirming the reliability of this approach. UMAP was performed on the first 5 principal components obtained from the top 1000 most variable genes in the hESC differentiation data (normalized, $\log _{2}$-transformed) before and after imputation (Supplementary Fig. 14) using the Seurat $\mathrm{R}$ package ${ }^{16}$. Cluster-specific markers were detected from the $\log _{2}$-transformed normalized data using Seurat. Detection rate was regressed out using the ScaleData function with vars.to.regress = nGene. Markers were detected with the FindAllMarkers function, using MAST26 test and setting logfc.threshold and min.pct to 0 , and min.cells.gene to 1 .

\section{GO term enrichment and transcription factor analyses}

All GO term enrichment analyses were performed with the topGO R package ${ }^{27}$. Enrichment in GO biological process terms among cluster-specific markers (Fig. 3C-D) was performed for each cell cluster and (no) imputation method separately, using as foreground the set of significant cluster markers detected by Seurat, with FDR $<0.05$ and $|\log \mathrm{FC}|>0.25$, and as background all genes in the Seurat result (both significant and non-significant). The classic algorithm was used, in combination with Fisher test, and $\log _{2}$ enrichment was quantified as the $\log _{2}$ of the ratio between the number of significant and expected genes in each term. Significantly enriched (p-value $<0.001$ and $\log _{2}$ enrichment > 0.5) GO biological process terms within each set of cluster markers, as detected in the original data (no masking, no imputation), were defined as "high confidence" terms.

For regulatory GO molecular function term enrichment analyses (Fig. 4A), significant (FDR $<0.05$ and $|\log \mathrm{FC}|>0.25)$ markers uniquely detected without / with each imputation method were combined across all clusters and tested for enrichment in the term "molecular function regulator" against the 
background of all genes obtained as the result of Seurat (both significant and non-significant). The classic algorithm was used, in combination with Fisher test, and $\log _{2}$ enrichment was quantified as the $\log _{2}$ of the ratio between the number of significant and expected genes in each term.

To identify transcription factors (TFs) among cluster markers exclusively detected using the network-based method, a curated TF list was downloaded from http://www.tfcheckpoint.org/index.php/browse.

\section{Determination of the optimal imputation method per gene}

In order to determine the best performing imputation method for each gene, $70 \%$ of the cells in each dataset were used as training data, where a percentage of the expression values were masked, as previously described. The remaining 30\% were used for testing. After masking, each of the tested imputation methods was applied to the training data and the imputed values of masked entries were then compared to the measured values. The weighted Mean Squared Error (MSE) was computed for each gene with masked entries:

$$
\frac{\text { avg }\left((\text { original-imputed })^{2}\right)}{\text { avg expression }+0.1}
$$

For each gene, the method leading to the smallest MSE was chosen as optimal.

\section{The ADImpute R package}

The ADImpute R package is composed of two main functions, EvaluateMethods and Impute. EvaluateMethods determines, for each gene, the method resulting in the lowest imputation error. Impute performs dropout imputation according to the choice of method provided by the user. Currently supported methods are scImpute, DrImpute, SCRABBLE, the Baseline and Network methods described in this manuscript and an Ensemble method, which takes the results from EvaluateMethods to select the imputation results from the gene-specific best method. Additionally, the user can choose to estimate the probability that each dropout value is a true zero, according to the approach used by scImpute, and leave the values unimputed if their probability of being a true zero falls above a user-defined threshold.

\section{Data and code availability}

The data used in this study are publicly available, as described in the Methods section. Human embryonic stem cell differentiation data are available in GEO under the accession number GSE75748. 
bioRxiv preprint doi: https://doi.org/10.1101/611517; this version posted June 10,2020. The copyright holder for this preprint (which was not certified by peer review) is the author/funder, who has granted bioRxiv a license to display the preprint in perpetuity. It is made available under aCC-BY-NC 4.0 International license.

Oligodendroglioma data are available in GEO under accession number GSE70630. Healthy human pancreas data are available in GEO under accession number GSE84133. The transcriptional regulatory network used in this study and the ADImpute $\mathrm{R}$ package are available from https://github.com/anacarolinaleote/ADImpute.

\section{References}

1. van Dijk, D. et al. Recovering Gene Interactions from Single-Cell Data Using Data Diffusion. Cell 174, 716-729.e27 (2018).

2. Li, W. V. \& Li, J. J. An accurate and robust imputation method scImpute for single-cell RNA-seq data. Nat Commun 9, 997 (2018).

3. Gong, W., Kwak, I.-Y., Pota, P., Koyano-Nakagawa, N. \& Garry, D. J. DrImpute: imputing dropout events in single cell RNA sequencing data. BMC Bioinformatics 19, 220 (2018).

4. Moussa, M. \& Măndoiu, I. I. Locality Sensitive Imputation for Single Cell RNA-Seq Data. J. Comput. Biol. 26, 822-835 (2019).

5. Andrews, T. \& Hemberg, M. False signals induced by single-cell imputation [version 2; peer review: 4 approved]. F1000Research 7, (2019).

6. Huang, M. et al. SAVER: gene expression recovery for single-cell RNA sequencing. Nat Methods 15, 539-542 (2018).

7. Meinshausen, N. \& Yu, B. Lasso-type recovery of sparse representations for high-dimensional data. http://dx.doi.org/10.21236/ada472998 (2006) doi:10.21236/ada472998.

8. Meinshausen, N. \& Bühlmann, P. Stability selection. J R Stat Soc Ser. B Stat Methodol 72, 417473 (2010).

9. Seifert, M., Friedrich, B. \& Beyer, A. Importance of rare gene copy number alterations for personalized tumor characterization and survival analysis. Genome Biol. 17, 204 (2016).

10. Chu, L.-F. et al. Single-cell RNA-seq reveals novel regulators of human embryonic stem cell differentiation to definitive endoderm. Genome Biol 17, 173 (2016).

11. Tirosh, I. et al. Single-cell RNA-seq supports a developmental hierarchy in human oligodendroglioma. Nature 539, 309-313 (2016).

12. Baron, M. et al. A Single-Cell Transcriptomic Map of the Human and Mouse Pancreas Reveals Inter- and Intra-cell Population Structure. Cell Syst 3, 346-360.e4 (2016).

13. Peng, T., Zhu, Q., Yin, P. \& Tan, K. SCRABBLE: single-cell RNA-seq imputation constrained by bulk RNA-seq data. Genome Biol. 20, 88 (2019).

14. Zhang, L. \& Zhang, S. Comparison of computational methods for imputing single-cell RNAsequencing data. (2017) doi:10.1101/241190.

15. McInnes, L., Healy, J., Saul, N. \& Grossberger, L. UMAP: Uniform Manifold Approximation and Projection. J. Open Source Softw. 3, 861 (2018).

16. Butler, A., Hoffman, P., Smibert, P., Papalexi, E. \& Satija, R. Integrating single-cell transcriptomic data across different conditions, technologies, and species. Nat Biotechnol 36, 411-420 (2018).

17. Vaquerizas, J. M., Kummerfeld, S. K., Teichmann, S. A. \& Luscombe, N. M. A census of human transcription factors: function, expression and evolution. Nat Rev Genet 10, 252-263 (2009).

18. Tugores, A. et al. The epithelium-specific ETS protein EHF/ESE-3 is a context-dependent transcriptional repressor downstream of MAPK signaling cascades. J Biol Chem 276, 20397-20406 (2001). 19. Boyd, C. A. R. Review: Epithelial aspects of human placental trophoblast. Placenta 34 Suppl, S24$6(2013)$. 
20. Tomaru, Y. et al. A transient disruption of fibroblastic transcriptional regulatory network facilitates trans-differentiation. Nucleic Acids Res. 42, 8905-8913 (2014).

21. Risso, D., Perraudeau, F., Gribkova, S., Dudoit, S. \& Vert, J.-P. A general and flexible method for signal extraction from single-cell RNA-seq data. Nat. Commun. 9, 284 (2018).

22. Barretina, J. et al. The Cancer Cell Line Encyclopedia enables predictive modelling of anticancer drug sensitivity. Nature 483, 603-607 (2012).

23. Klijn, C. et al. A comprehensive transcriptional portrait of human cancer cell lines. Nat. Biotechnol. 33, 306-312 (2015).

24. Leek, J. T. \& Storey, J. D. Capturing Heterogeneity in Gene Expression Studies by Surrogate Variable Analysis. PLOS Genet. 3, e161 (2007).

25. Talwar, D., Mongia, A., Sengupta, D. \& Majumdar, A. AutoImpute: Autoencoder based imputation of single-cell RNA-seq data. Sci. Rep. 8, 16329 (2018).

26. Finak, G. et al. MAST: a flexible statistical framework for assessing transcriptional changes and characterizing heterogeneity in single-cell RNA sequencing data. Genome Biol. 16, 278-278 (2015).

27. Alexa, A., Rahnenführer, J. \& Lengauer, T. Improved scoring of functional groups from gene expression data by decorrelating GO graph structure. Bioinformatics 22, 1600-1607 (2006).

\section{Acknowledgements}

The results here shown are in part based upon data generated by the TCGA Research Network: http://cancergenome.nih.gov/. The Genotype-Tissue Expression (GTEx) Project was supported by the Common Fund of the Office of the Director of the National Institutes of Health, and by NCI, NHGRI, NHLBI, NIDA, NIMH, and NINDS. The data used for the analyses described in this manuscript were obtained from the GTEx Portal on 12/2019. A.C.L. received support by the Cologne Graduate School of Ageing Research. X.W. received financial support from the National Natural Science Foundation of China (61871463) and Natural Science Foundation of Fujian Province of China (2017J01068).

We gratefully acknowledge help from Dr. Michael Seifert (TU Dresden, Germany) on the construction of the transcriptional regulatory network.

\section{Author Contribution}

$A B$ envisioned the study. XW implemented and performed the network training and testing. ACL implemented and tested the dropout imputation method. All authors contributed to the writing of the manuscript.

\section{Competing Interests}

The authors declare no competing interests. 